\title{
Multi-Objective Optimization of A HVAC System: Non-Dominated Sorting-Based Differential Evolution Approach
}

\author{
${ }^{1}$ Y.N. Kuan and ${ }^{2}$ H.S. Ong \\ ${ }^{1}$ Faculty of Engineering and Technology, Tunku Abdul Rahman University College, \\ Kuala Lumpur, Malaysia \\ ${ }^{2}$ College of Engineering, University of Tenaga Nasional, Kajang, Malaysia
}

\begin{abstract}
Energy efficiency of Heating Ventilating and Air Conditioning (HVAC) systems plays an important role in reducing the world's energy needs. Optimization of HVAC system is one of the promising ways to improve energy efficiency and to help in slowing down the depletion of our energy resources. This study proposed an improved version of multi-objective optimization algorithm which integrates the simple yet powerful differential evolution approach to the popular non-dominated sorting Genetic algorithm-II in solving for optimization of a HVAC system. Energy consumption and thermal comfort are the two conflicting objectives to be optimized with hourly cooling temperature set points of the thermal zone serve as the design variables of the optimization. Optimization is performed through simulation of a case study using MATLAB coupled with EnergyPlus Software. Cardinality, space and hyper volume metrics are used to measure and compare the quality of the Pareto fronts obtained by the proposed algorithm, NSDE with the base algorithms, Differential Evolution (DE) and Non-nominated Sorting Genetic Algorithm- $\Pi$ (NSGA-I). Decision making is also performed to evaluate the energy performance of the proposed algorithm. Simulation results demonstrated that the NSDE produces better quality Pareto optimal solutions compared to DE and NSGA-II as well as better energy saving capability of the algorithm.
\end{abstract}

Key words: Energy, thermal comfort, optimization, multi-objective, promising, Non-dominating Sorting-based Differential Evolution (NSDE)

\section{INTRODUCTION}

Located near the equator, Malaysia's climate is regarded as hot and humid throughout the year. Heating Ventilating and Air Conditioning (HVAC) systems have become a necessity in providing comfortable thermal condition in building indoor spaces. As a consequence, energy consumption of air conditioners has increased to more than $60 \%$ of total building energy in Malaysia according to an audit carried out by Malaysia green technology corporation. With mushrooming of new properties and building usage in urban area, the energy demand for air-conditioning is expected to raise even higher in the near future. Therefore, reducing energy consumption of HVAC systems is very crucial for conservation and sustainability of energy. Research and studies by scientists and scholars reveal that optimization of HVAC systems is one of the most effective ways to conserve energy. Optimized HVAC system is shown to benefit in energy reduction of $7-30 \%$ by Nassif et al. (2004), Fong et al. (2006), Mossolly et al. (2009) and Hussain et al. (2014).
In mathematical aspect, optimization is the selection of a best element from some set of available alternatives with regard to certain objective or objectives (Chiandussi et al., 2012). Since HVAC system is an integration of many complicated parts such as heat exchanger, blower, condenser coil, evaporator coil, thermostat, etc., HVAC optimization thus involves searching of the best possible operating points of these parts under several dynamic conditions such as outdoor weather and indoor loads with the objectives of maximizing the occupants thermal comfort level and minimizing the energy costs of the system. This searching process, once was thought to be time and memory space consuming has evolved to be one of the promising way to solve complex engineering problem, thanks to the advancement of computing technology. Researchers think that most of the energy savings can be achieved through optimum control of various components in HVAC system.

Research on HVAC systems optimization can be seen as early as by Zheng and Zaheer-Uddin (1996), Huang and Lam (1997) using Sequential Quadratic Programming

Corresponding Author: Y.N. Kuan, Faculty of Engineering and Technology, Tunku Abdul Rahman University College, Kuala Lumpur, Malaysia 
(SQP) and Genetic Algorithm (GA), respectively for HVAC Proportional-Integral (PI) control, though occupant thermal comfort was not the criteria in their optimization. Since then, there have been increasing trend of studies focusing on optimization of HVAC systems, most with the aims of maintaining the indoor thermal comfort of occupants and indoor air quality but with minimum energy spending possible. Since, HVAC is a complex and nonlinear system that involves hundreds of variables, meta-heuristic and stochastics based optimization seem to be more preferred by most researchers in recent years. These include evolutionary algorithms (such as Genetic Algorithm (GA), Evolution Strategies (ES) and Evolution Programming (EP)) and bio-inspired optimizations (such as Particle Swarm intelligence (PSO), Ant colony, Bat Algorithm (BA)).

Wang and Jin (2000), Xu and Wang (2009) used GA to perform optimal control of Variable Air Volume (VAV) air conditioning system by simultaneously searching for three HVAC parameters: supply air temperature, chilled water temperature and outdoor ventilation rate. Mossolly et al. (2009) used GA to search for the optimal supply air temperature and supply air flow rate by maintaining the Predicted Mean Vote (PMV) and Indoor Air Quality (IAQ) of multi-zone air conditioning system. Hussain et al. (2014) incorporated GA in the Fuzzy Logic Controller (FLC) dedicated to control of HVAC systems that concerning energy efficiency and thermal comfort requirement. GA is also extended to solve multi objective problems. Wright et al. (2002) employed Multi-Objective Genetic Algorithm (MOGA) to search for the optimum sizing of HVAC system, simultaneously with the optimization of its supervisory control strategy to solve two conflicting objectives regarding system energy use and occupant comfort. Nassif et al. (2004) evaluated both Non-dominated Sorting Genetic Algorithm (NSGA) and NSGA-II for two-objective optimization by tuning four HVAC parameters concurrently. NSGA-II is very well-known as a fast and elitist multi-objective GA. Other variants of GA include Multi-Phase Genetic Algorithm (MPGA) by Beghi et al. (2011) for optimization of multiple chiller systems and Multi-Island Genetic Algorithm (MIGA) by Seo et al. (2014) for optimal operation of HVAC system in an apartment house.

Some scholars Kusiak et al. (2010), Yang and Wang (2012), He et al. (2014) used well-known bio-inspired swarm intelligence algorithm such as Particle Swarm intelligence (PSO) to find different optimal control set points that applied on different parts of the HVAC system. Beghi et al. (2012) employed PSO to minimize the overall energy consumption of multiple chiller systems by determining the load fraction that each chiller has to satisfy. Kusiak et al. (2011) applying Strength Multi-Objective Particle Swarm Optimization (S-MOPSO) to a scalarized three-objectives optimization of a predictive Air Handling Unit (AHU) system developed using feedforward neural network. Zeng et al. (2015) used Firefly Algorithm (FA) to search for the optimal supply temperature set point and supply air static pressure set point of a multi-zone HVAC system whereas (Coelho and Mariani, 2013) used Improved Firefly Algorithm (IFA) to minimize energy consumption of multi-chiller system by determining the part load ratio of each chiller. The same researcher later investigated the same multiple chillers optimization problem using Differential Bat Algorithm (DBA) (Coelho and Askarzadeh, 2016). Other literature for optimization of HVAC systems include Evolutionary Programming (EP), Evolution Strategies (ES) and Differential Evolution (DE) by Fong et al. (2006, 2009), Kusiak et al. (2010) and Lee et al. (2011). All of these approaches are showing promising and satisfactory result in optimization of HVAC systems. From the abovementioned review, it can be concluded that multi-objective optimization approach is a feasible method to search for optimal control of HVAC systems for energy efficiency improvement.

The current study proposed an improved version of multi-objective optimization algorithm based on two promising optimization approaches, Non-dominated Sorting Genetic Algorithm-II (NSGA-II) and Differential Evolution(DE). The aims of the optimization is to minimize the daily energy consumption of a HVAC system in a single zone building and at the same time maximizing the indoor thermal comfort of the space. The hourly cooling temperature set points of the HVAC system is optimized and the performance of the proposed algorithm would be compared with the original DE, NSGA-II and a baseline scenario.

\section{MATERIALS AND METHODS}

Multi objective optimization: HVAC systems often deal with two major opposing design objectives: maximize the thermal comfort of the occupants at the same time minimize the power consumption or energy cost. Thus, Multi-Objective Optimization (MOOP) is usually being applied in HVAC control systems than single objective optimization. In MOOP, enhancement of one objective always leads to degradation of another. In this scenario, a trade-off must be created. Generally, a multi-objective optimization problem can be represented by: 
Minimize $f_{i}(x) \quad(i=1,2, \ldots, M)$

s. t. $x \in X$

where, $\mathrm{M} \geq 1 . \mathrm{X}$ is the feasible set of decision vectors. The feasible set is usually bound by some constraint functions. Multi-objective optimizations search for sets of best trade-off solutions in the objective space which is called Pareto optimal. Pareto optimal is the solutions that cannot be improved in any of the objectives without degrading at least one of the objectives (Awad and Khanna, 2015). A solution $x_{1} \in X$ is said to dominate another solution $\mathrm{x}_{2} \in \mathrm{X}$ if:

$$
\begin{aligned}
& f_{i}\left(x_{1}\right) \leq f_{i}\left(x_{2}\right) \text { for all indices } i \in\{1,2, \ldots, k\} \\
& f_{j}\left(x_{1}\right)<f_{j}\left(x_{2}\right) \text { for at least one index } j \in\{1,2, \ldots, k\}
\end{aligned}
$$

Many researchers performed scalarization to multi-objective HVAC optimization(Huang andLam, 1997; Wang and Jin 2000; Mossolly et al., 2009) which trans form the multi-objective problem into single objective problem by allocating different weight factors to the objective functions:

$$
\underset{\mathrm{x} \in \mathrm{X}}{\operatorname{Minimize}} \sum_{\mathrm{i}=1}^{\mathrm{n}} \mathrm{w}_{\mathrm{i}} \mathrm{f}_{\mathrm{i}}(\mathrm{x})
$$

where, $\mathrm{w}_{\mathrm{i}}$ is the weight factors of the objective functions. The weight factors can be set according to occupants' preferences. This is particularly useful when the preference factor of the objectives is known in advance. Another approach used is the true multi objective optimization where a set of Pareto optimal solutions are produced. In this regards, some of the metaheuristic single-objective optimization algorithms such as GA, NSGA-II, PSO and DE have their multi-objective version developed. Nassif et al. (2004) optimized two objective functions simultaneously using NSGA-II to find the optimal settings of supply air temperature, supply duct static pressure, chilled water temperature and zone temperature to give the minimum energy usage and maximum PMV. Magnier and Haghighat (2010) too optimized thermal comfort and energy consumption using NSGA-II by searching for optimal heating set points, cooling set points, relative humidity set points, supply air flow rates and thermostat delays. Wright et al. (2002) optimized energy cost and occupant thermal discomfort using MOGA by adjusting supply air temperature and supply air flow rate. For this approach, many Pareto optimal solutions are produced and all solutions are considered equally good in satisfying the objective functions. It then depends on the user's preferences or prioritization in choosing for the best solution from the Pareto set and this system is called Multi-Criteria Decision Making (MCDM).

\section{Optimization algorithm}

Non-dominated Sorting-based Differential Evolution (NSDE): Non-dominated Sorting Genetic Algorithm II (NSGA-II) is a multi-objective genetic algorithm originally proposed by Deb et al. (2002). It is the improved version of Non-dominated Sorting Genetic Algorithm (NSGA) proposed earlier by Srinivas and Deb (1994). NSGA-II is a very well-known population based multi-objective optimization algorithm with two special features implemented in its searching operation, non-dominated sorting and crowding distance. In non-dominated sorting, the population is sorted based on non-domination and categorized into different ranking of fronts. Crowding distance is then assigned to find the Euclidean distance between each front population, so that, a better diversity can be obtained. Selections are then performed based on the non-dominated rank and crowding distance.

On the other hand, Differential Evolution (DE) is a population based evolutionary algorithm which is originally proposed by Storn and Price (1997). DE is very simple, yet very powerful and useful in solving real parameter optimization problems with very good performance as reported by Lee et al. (2011). This algorithm uses simple mutation operator based on difference of solution vectors to create new candidate solutions. The new candidate solution is then competes against its corresponding parent and replaces the parent if the candidate solution has a higher fitness value.

Both NSGA-II and DE are population-based metaheuristic evolutionary algorithm which contains the four main operators: initialization, mutation, crossover and selection, except that the sequence of the operations are different. Since, NSGA-II adopts the conventional binary crossover and polynomial mutation, the crossover and mutation procedure are complex, slower in speed and tend to trap into local optima. On the other hand, DE uses a greedy search and thus is less stochastic compared to other optimization algorithms. Therefore, an improved version of optimization is proposed.

In this proposed research, optimization of HVAC system is implemented using Non-dominated Sorting-based Differential Evolution (NSDE) which uses NSGA-II as the main framework. Here, elitism of non-dominated sorting and diversity of crowding distance in NSGA-II are preserved with differential mutation and crossover of $\mathrm{DE}$ replace the genetic operators in conventional NSGA-II. DE controls the behaviour of 


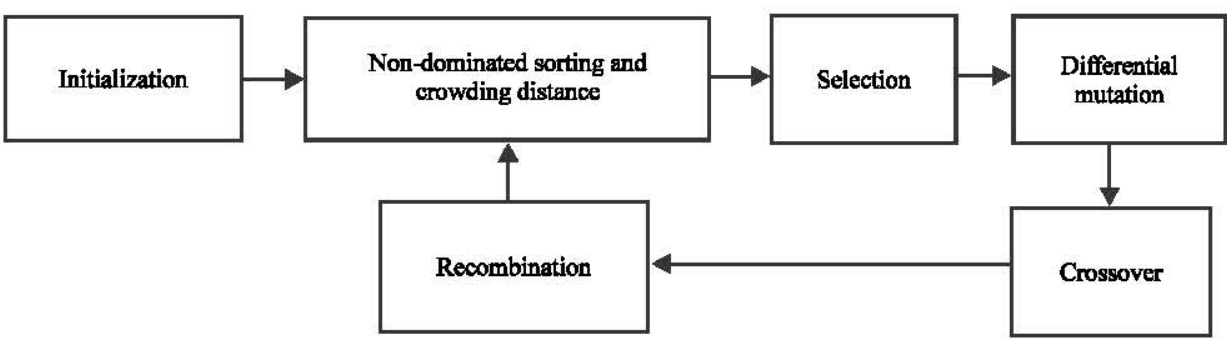

Fig. 1: Main operation of Non-dominated Sorting-based Differential Evolution (NSDE)

mutation operator with a flexible distribution which made it much simple and straight forward to implement. The main operations of NSDE is shown in Fig. 1 and are described as:

Initialization: Initial populations are generated randomly based on problem range and constraints if any. Initial populations act as the parent populations $\left(\mathrm{x}_{\mathrm{G}}\right)$ at the first generation.

Non-dominated sort: Objective functions of each initialized population is evaluated and the population is sorted and ranked based on non-domination level with 1 the best level, 2 the second best level and so on.

Crowding distance: This operation aims to get an estimation of the density of solutions surrounding a particular solution. Here, the Euclidean distance between each individual in the front population is determined. All individuals in the front population is assigned with a crowding distance value. The larger the value, the better the diversity.

Selection: Selection is done by binary tournament. Parents are selected from the population pool for reproduction based on non-dominated rank and crowding distance. An individual population is selected if the rank is lesser than the other. If it has the same rank as other, then individual with greater crowding distance value is selected.

Genetic operator-mutation and crossover: Here, the genetic operator of NSGA-II is replaced by the simple yet efficient differential evolution mutation and crossover operator. Besides the typical parameters used in evolutionary algorithms such as number of population, dimensions and number of generation, $\mathrm{DE}$ adopts two other parameters, cross over probability, CR and scaling factor, F. CR (value is between 0 and 1) controls the influence of parent in generation of new candidate solutions (offspring). Lower value indicates higher influence of the parent in the features of its offspring. $F$ (value is between 0 and 1) scales the influence of pairs of solutions selected to calculate the mutation value in mutation operation (Mezura-Montes et al., 2008).

Mutation: In mutation, new solutions $\left(\mathrm{v}_{\mathrm{G}}\right)$ are created by adding the mutation differential of a pair of randomly chosen solutions $\left(\mathrm{x}_{\mathrm{r} 1, \mathrm{G}}\right.$ and $\left.\mathrm{x}_{\mathrm{r} 2, \mathrm{G}}\right)$ with a donor solution $\left(\mathrm{x}_{\mathrm{r} 3, \mathrm{G}}\right)$. The difference of $\mathrm{x}_{\mathrm{r} 1, \mathrm{G}}$ and $\mathrm{x}_{\mathrm{r} 2, \mathrm{G}}$ is scaled with parameter $\mathrm{F}$, after which it is added to the donor solution $\left(\mathrm{X}_{\mathrm{r} 3, \mathrm{G}}\right)$ that is either chosen at random or selected from the best solution in the population. In this proposed research, the most popular $\mathrm{DE}$ variant $(\mathrm{DE} / \mathrm{rand} / 1 / \mathrm{bin})$ is adopted whereby random donor solution is selected for the mutation operation. The new solutions are also called mutation vector. The process can be expressed as:

$$
\mathrm{v}_{\mathrm{G}}=\mathrm{x}_{\mathrm{r} 3, \mathrm{G}}+\mathrm{F}\left(\mathrm{x}_{\mathrm{r} 1, \mathrm{G}}-\mathrm{x}_{\mathrm{r} 2, \mathrm{G}}\right)
$$

Crossover: To improve the diversity of population, crossover operation is performed. Here, the mutation vector $\left(\mathrm{v}_{\mathrm{G}}\right)$ is crossovered with original parent $\left(\mathrm{x}_{\mathrm{G}}\right)$ with either binomial or exponential method. In this proposed research, binomial method is adopted. Crossover is performed to produce offspring $\left(\mathrm{u}_{\mathrm{G}}\right)$ whenever a randomly generated number between 0 and 1 is less than the parameter CR. The operation can be outlined as:

$$
\mathrm{u}_{\mathrm{G}}=\left\{\begin{array}{l}
\mathrm{v}_{\mathrm{G}}, \text { if }(\operatorname{rand}[0,1]<\mathrm{CR}) \\
\mathrm{x}_{\mathrm{G}}, \text { otherwise }
\end{array}\right.
$$

Recombination: Objective functions of offspring population $\left(\mathrm{u}_{\mathrm{G}}\right)$ are evaluated. The offspring population, $\mathrm{u}_{\mathrm{G}}$ are then recombined with the current generation population, $\mathrm{x}_{\mathrm{G}}$ (parents in the first generation) to produce 
(a)

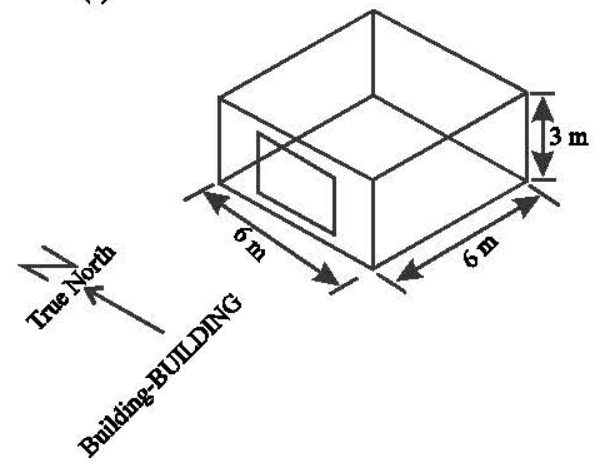

(b)

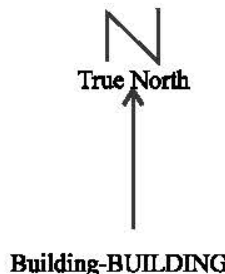

Building-BUILDING

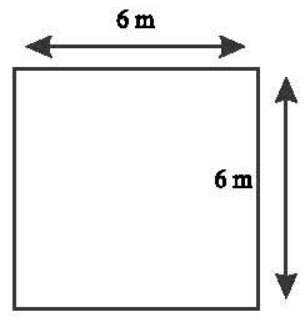

Fig. 2: a) Architecture schematic view of the single thermal zone and b) Top view of the single thermal zone

$\mathrm{r}_{\mathrm{G}}$ and hence, two times population size is produced. Here, non-dominated sorting is performed on the combined population and only the best are passed to the next generation to ensure elitism. The process then repeats until the termination criteria is reached. The termination criteria can be determined by number of maximum iterations or by function tolerance. In other words, iterations end when the pre-set maximum iterations value is reached or when the fitness function does not vary more than the pre-set tolerance value over consecutive iterations.

\section{Simulation}

Case study model: The proposed algorithm is evaluated using a single zone in a building which is modelled using EnergyPlus Software. The evaluated building is located in capital city of Malaysia, Kuala Lumpur with latitude of $3.12^{\circ}$, longitude of $101.55^{\circ}$ and elevation of 22 meter above sea level. The single thermal zone has a square geometry of $108 \mathrm{~m}^{3}$ with length, width and ceiling height of $6 \times 6 \times 3$ meter, respectively. There is a window of $3 \times 2$ meter facing the west. The thermal zone is simulated with the north wall, west window and roof exposed to the Sun and wind while the east and south wall are internal walls adjacent to other thermal zones in the same building. Figure 2 shows the architecture schematic view of the single thermal zone. The single zone is simulated with six persons occupy the space from 7 a.m. to 5 p.m. in weekday with sedentary activities such as reading, writing, typing and having meeting (150 W/person). The occupants are assumed to be in typical office clothing such as trousers and long sleeved shirts $(\approx 0.6$ clo). The air velocity of the zone is assumed to be fix at $0.25 \mathrm{~m} / \mathrm{sec}$ to comply with the ASHRAE 55 standards for summer season (ANSI/ASHRAE., 2013; Anonymous, 2013). The single zone is equipped with a packaged terminal air conditioner system that provides cooling mode only,

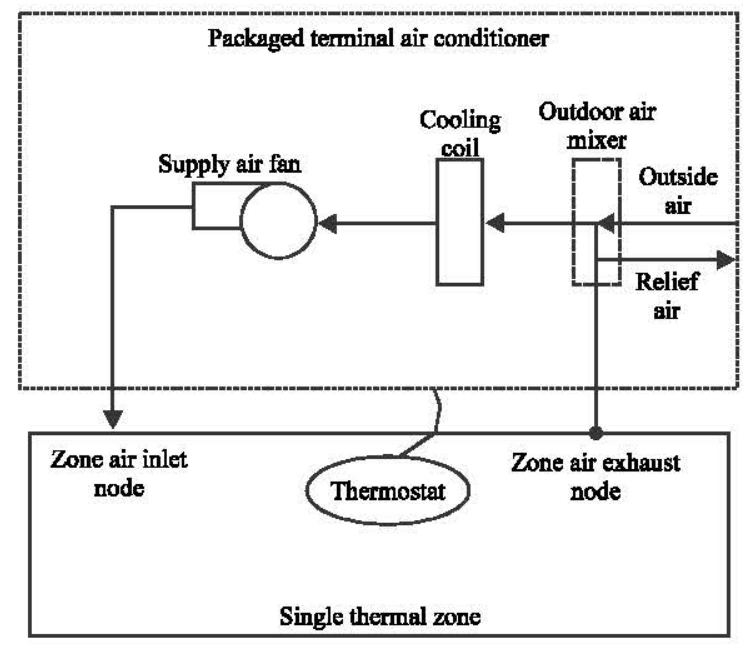

Fig. 3: Schematic of a packaged terminal air-conditioner system

since, Malaysia is a tropical country with hot and humid weather all year long. Simulation is run using the measured weather data for the month of May as this is the hottest month in the year according to the weather source by EnergyPlus. Figure 3 shows the schematic diagram of a packaged terminal air-conditioner system modelled using EnergyPlus used for the proposed optimization. The zone temperature is controlled using the single thermostat in the air-conditioner system.

Objective function and design variables: In this research, Non-dominated Sorting Differential Evolution (NSDE) is used to optimize the HVAC system of a single zone thermal building, taking into consideration the energy performance and also the thermal comfort of the occupants. The optimization has two-objective functions: 
- $\mathrm{f}_{1}(\mathrm{x})$ : minimize the energy consumption

- $\mathrm{f}_{2}(\mathrm{x})$ : minimize the maximum Predicted Percentage of Dissatisfied (PPD max)

For each day:

$$
\begin{aligned}
& \text { Minimize } F(x)=\left[f_{1}(x), f_{2}(x)\right] \\
& \text { Subject to } x_{1} \leq x \leq x_{u}
\end{aligned}
$$

where, $x_{1}$ and $x_{u}$ are the lower bound and upper bound of the design variables. The first objective function to be minimized is the total energy consumption of the air-conditioner system in 24 hours. In this research, even though the zone is simulated with the presence of lights and electric equipment, only electric power of the air-conditioner is to be optimized.

Second objective function is regarding occupants' thermal comfort. Predicted Mean Vote (PMV) index is commonly used to measure the condition of mind that expresses satisfaction with thermal environment. It is calculated using Fanger's equation (ASHRAE, 2009). It depends on environmental parameters such as air temperature, humidity, mean radiant temperature, air velocity and personal variables such as metabolic rate and clothing insulation. PMV is associated with thermal sensation scale that runs from cold $(-3)$ to hot $(+3)$ with 0 as the ideal value. The recommended acceptable PMV range for thermal comfort from ASHRAE Standard 55 is between -0.5 to 0.5 for indoor spaces (ASHRAE, 2009).

Although predicting thermal sensation is an important measure in determining a comfortable condition, it is more meaningful and beneficial to consider the thermal satisfaction of occupants. Predicted Percentage of Dissatisfied (PPD) is another equation developed by Fanger and it is a function of PMV as shown in Fig. 4. PPD is an index to predict the percentage of occupants that will be dissatisfied with the thermal conditions. As PMV value moves away from zero or neutral, PPD increases. As we can see from Fig. 4 a PMV range of \pm 0.5 which is the recommended PMV by ASHARE corresponds to a PPD of $10 \%$. In this proposed research, $\mathrm{PPD}(\%)$ is used as the thermal comfort indicator instead of PMV to better reflect the minimization of the objective function in this optimization. Besides that, maximum PPD $\left(\mathrm{PPD}^{\mathrm{max}}\right)$ is optimized instead of average PPD $\left(\mathrm{PPD}^{\text {ave }}\right)$ to ensure the evaluated PPD are kept at a low value all the time throughout the examined day.

Next, the design variables to be optimized are the hourly cooling temperature set points of the single zone during working hours (from 7 a.m. to 5 p.m.) thus resulting in ten design variables in a simulation day. The cooling set point is fix at $26^{\circ} \mathrm{C}$ from time 0.00 to $7: 00$ and from $17: 00$ to $24: 00$ as the occupancy is set to zero assuming no one

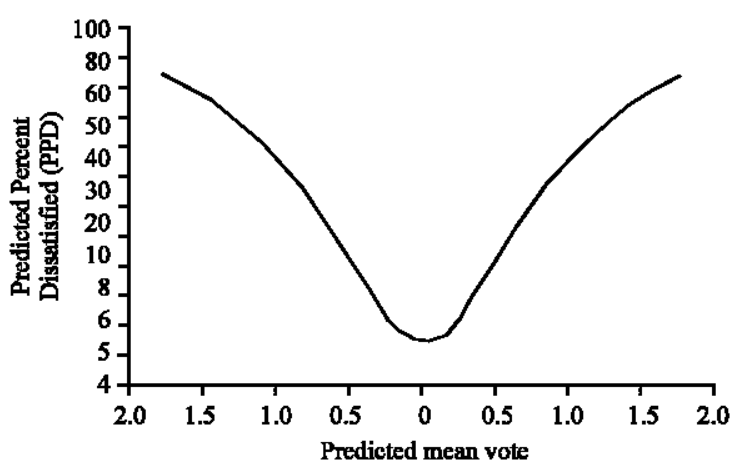

Fig. 4: Predicted Percentage of Dissatisfied (PPD) as a function of Predicted Mean Vote (PMV), (ASHRAE, 2009)

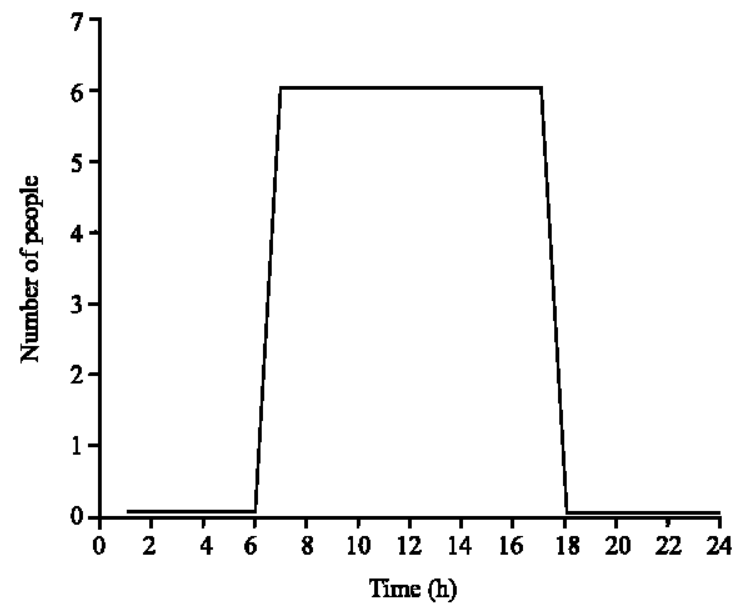

Fig. 5: Occupancy model of the simulated day

is present in the zone at this period of time. Figure 5 shows the occupancy model of the simulated day. The constraints of the design variable is set to be between 16 to $26^{\circ} \mathrm{C}$. The cooling set points are to be controlled using the thermostat in the single thermal zone.

Coupling of MATLAB and EnergyPlus for HVAC optimization: The proposed multi-objective optimization of HVAC system using Non-dominated Sorting-based Differential Evolution (NSDE) is implemented and simulated using MATLAB, a powerful and flexible optimization software. On the other hand, EnergyPlus a comprehensive building simulation software is used to create the building, HVAC system and occupancy model of our simulation. Besides that, EnergyPlus can be used to accurately calculate the building electricity consumption and thermal comfort of occupants in the simulated zone. The optimization procedure and iterations are programmed and run using MATLAB environment. 


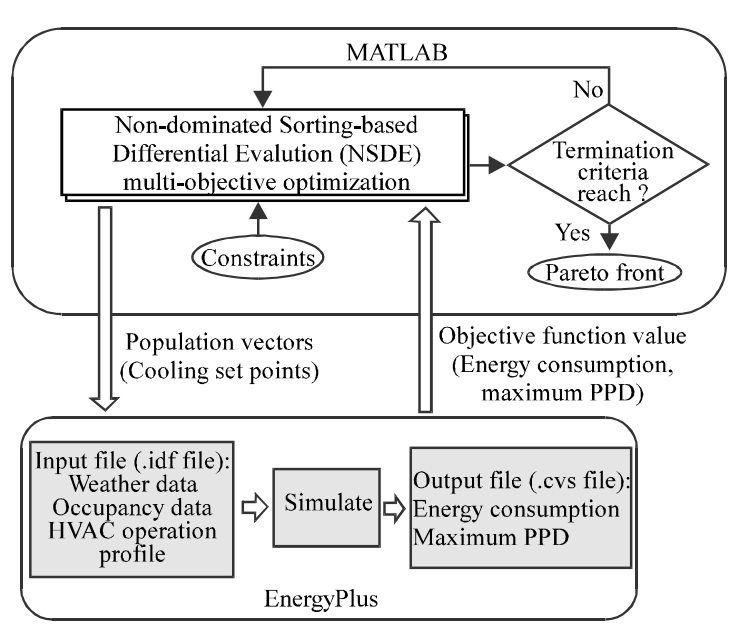

Fig. 6: Coupling of MATLAB and EnergyPlus framework

MATLAB code is written to invoke EnergyPlus which is treated as an external software, each time objective function evaluation is performed. In other words, EnergyPlus works as a black box function evaluator when validated data such as weather file, HVAC and occupancy profile are input to the system. Figure 6 shows the coupling of MATLAB and EnergyPlus framework in this proposed research.

Some researchers perform coupling of MATLAB and EnergyPlus with the aid of third party software such as JEPlus and MLE+ (Xu et al., 2013; Garnier et al., 2015 and Delgarm et al., 2016). The novelty of this research is for MATLAB, the main optimization engine to control and communicate with EnergyPlus without any unnecessary third party interface. This greatly reduce the complexity of the whole simulation and optimization system.

\section{RESULTS AND DISCUSSION}

Parameter setting: Simulation is carried out for one working day ( 24 hours) to determine the optimal solutions of the HVAC system using our proposed Non-dominated Sorting-based Differential Evolution (NSDE) as explained in previous section. For comparison purposes, optimization are also performed using Differential Evolution (DE) and Non-dominated Sorting Genetic Algorithm- $\Pi$ (NSGA- $\Pi$ ). Simulation of various algorithms are run for multiple times using different control parameters and they are found to yield the best results with the parameter setting as shown in Table 1.

The selected simulation day is on 2 May as it falls on the hottest month in the year to better justify the efficiency of different algorithms in the extreme weather of a tropical country. Figure 7 shows the outdoor temperature of the simulated day according to weather file sourced by EnergyPlus. All algorithms are configured with the same number of population size, $\mathrm{N}$ which is 40 and maximum iterations of 50 which also serves as the stopping criteria for the optimization.

Experimental results: Results of the simulation is shown in Fig. 8. Here, the Pareto fronts or the best optimal solutions of the three multi-objective optimization algorithms are presented. As would be expected, decrease in thermal discomfort (PPD) requires an increase in energy consumption. The trend lines can be observed in all the multi-objective approaches involved.

The performance and quality of the Pareto fronts obtained are evaluated using some common performance metrics. Suitable performance metrics should be chosen as we are dealing with practical real-world application where the true Pareto fronts are unknown. First, Riquelme et al. (2015) in measure for cardinality which refers to number of non-dominated solutions obtained from an optimization run, algorithm that produces larger number of solutions is intuitively preferred (Riquelme et al., 2015). In this case, NSGA-II has the highest score with 39 non-dominated solutions found, followed by the proposed NSDE with 33 solutions and finally DE with only 5 optimal solutions. Even though the proposed NSDE has lesser number of non-dominatedsolutions compared to NSGA-II, the number of solutions is still notably high (Table 2).

Next, the Pareto solutions are assessed for its diversity. Here, Spacing metric (S) is used to evaluate how uniformly the solutions are distributed in the objective space. It measures the variance of the distance of each solution to its nearest neighbour. An algorithm which produces Pareto solutions that are evenly distributed has smaller spacing value and is better (Tydrichova and Kozlowska, 2017). Table 2 shows the spacing metric of the algorithms examined. Clearly, the proposed NSDE gives the best spacing measure among the three. NSGA-II has disconnected Pareto fronts thus, yields a poorer result.

Another important metric used to evaluate the Pareto-optimal fronts is Hyper-Volume (HV) metric. HV metric provides a qualitative measure of convergence as well as divergence in a combined manner. It calculates the volume in the objective space covered by a reference point and the Pareto fronts obtained. Theoretically, for each Pareto solution, a hypercube is constructed using a reference point and the corresponding Pareto point in forming the diagonal corners of the hypercube. The reference point can be obtained using a vector of worst 
Table 1: Parameter setting for different optimization algorithms

\begin{tabular}{lccccc}
\hline Varibles & Crossover probability & Scaling factor & Distribution index for crossover & Distribution index for mutation & Mutation probability \\
\hline DE & 0.1 & 0.5 & - & - & - \\
NSGA-I & - & - & 20 & 20 & 0.1 \\
NSDE (proposed) & 0.1 & 0.5 & - & - & - \\
\hline
\end{tabular}

Table 2: Cardinality metric of all algorithm (Larger value is better) (Riquelme et al., 2015)

\begin{tabular}{lc}
\hline Algorithms & Number of non-dominated solutions \\
\hline DE & 5 \\
NSGA-II & 39 \\
NSDE (proposed) & 33 \\
\hline
\end{tabular}

Table 3: Spacing metric (S) of all algorithms (Smaller value is better) (Tydrichova and Kozlowska, 2017)

\begin{tabular}{lc}
\hline Algorithms & Spacing metric (S) \\
DE & 22.2686 \\
NSGA-II & 11.5230 \\
NSDE (proposed) & 6.1775 \\
\hline
\end{tabular}

Table 4: Hypervolume metric (HV) of all algorithm (Larger value is better) (Riquelme et al., 2015

\begin{tabular}{lc}
\hline Algorithms & Hypervolume metric (HV) \\
\hline DE & $2.7618 \times 10^{4}$ \\
NSGA-II & $2.9432 \times 10^{4}$ \\
NSDE (proposed) & $3.1723 \times 10^{4}$ \\
\hline
\end{tabular}

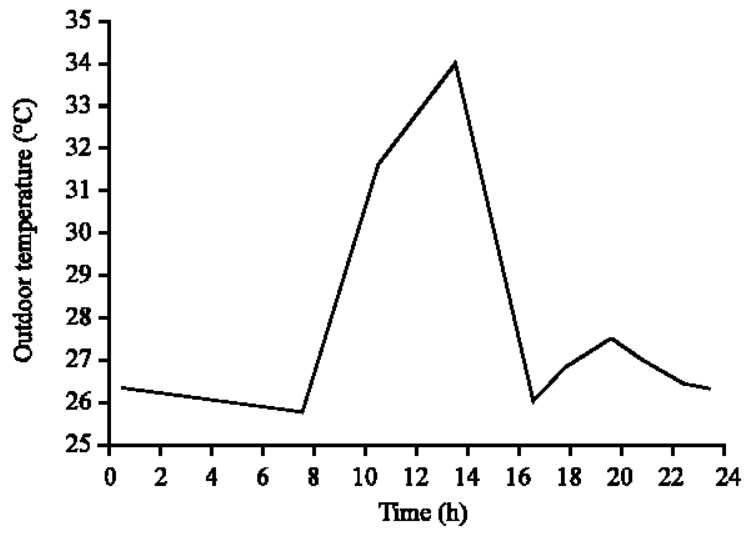

Fig. 7: Outdoor temperature profile of the simulated day

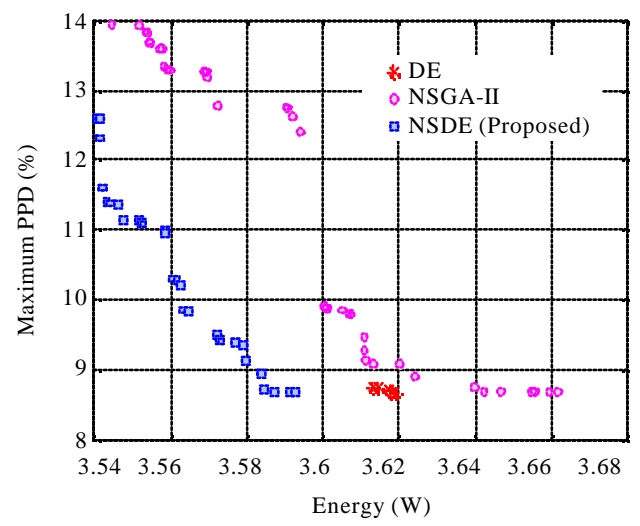

Fig. 8: Pareto fronts of DE, NSGA-II and NSDE optimization objective function values ("Multi-objective performance metrics"). An algorithm with larger hypervolume value are better. HV metric is very much recommended as this performance indicator does not require knowledge of true Pareto fronts. Table 4 shows the hypervolume value calculated based on a common reference point in the objective space for all three algorithms. The proposed NSDE yields the best $\mathrm{HV}$ result which indicates that it has a better explorative behaviour and offers more diversity of solutions compared to DE and NSGA-II (Table 3).

Decision making: After the complete set of Pareto optimal solutions are found, Decision Making (DM) is performed to select one of the preferred non-dominated solutions for energy consumption calculation. Generally, logical decision making depends on knowledge from experts or specialists of related field that involves analysis and diagnosis of the system. However, in most of the situation, DM could be naturally intuitive and depends on preferences, interests and experiences of the users. In this research, decision making is done based on different preferences on objective function $\mathrm{f}_{2}$ value which is the maximum Predicted Percentage of Dissatisfied ( $\mathrm{PPD}^{\mathrm{max}}$ ). Energy consumption that correspond to the selected PPD is examined and compared among all the optimization approaches. For every configuration, the results are benchmarked with a baseline scenario where no optimization is involved. For baseline scenario, the cooling temperature set point is fix at $22^{\circ} \mathrm{C}$ from 7 a.m. to 5 p.m. It is to be reminded that for all the optimization algorithms in this simulation research, cooling temperature set points are set to a constant value of $26^{\circ} \mathrm{C}$ from time 0.00 to $7: 00$ and from $17: 00$ to $24: 00$ as the occupancy is assumed to be zero at these hours.

In the first configuration, $\mathrm{PPD}^{\max }$ of below $10 \%$ is selected for energy performance evaluation. A PPD of $10 \%$ correspond to PMV range of \pm 0.5 which is the PMV limits recommended by ASHARE standard 55 (ASHRAE, 2009). As such, Pareto point right below $\mathrm{PPD}^{\max }$ of $10 \%$ is chosen as our solution for decision making, so that, an allowable thermal comfort is attained while energy consumption can be minimized. The selected solution for each algorithm is highlighted in Fig. 9. With condition of $\mathrm{PPD}^{\max }$ not exceeding $10 \%$, energy consumption of DE, NSGA-II and NSDE are recorded to be $36.13,36.01$ and $35.64 \mathrm{~kW}$, respectively. This corresponds to energy reduction of $6.33,6.64$ and $7.60 \%$, 


\begin{tabular}{|c|c|c|c|c|c|c|}
\hline \multirow[b]{2}{*}{ Variables } & \multicolumn{2}{|c|}{ PPD below $10 \%$} & \multicolumn{2}{|l|}{ Lowest PPD } & \multicolumn{2}{|l|}{ Highest PPD } \\
\hline & Energy $(\mathrm{kW})$ & Diff. (\%) & Energy $(\mathrm{kW})$ & Diff. $(\%)$ & Energy $(\mathrm{kW})$ & Diff. $(\%)$ \\
\hline Baseline & 38.57 & - & 38.57 & -38.57 & - & \\
\hline $\mathrm{DE}$ & 36.13 & -6.33 & 36.19 & -6.17 & 36.13 & -6.33 \\
\hline NSGA-II & 36.01 & -6.64 & 36.62 & -5.06 & 35.45 & -8.09 \\
\hline NSDE (proposed) & 35.64 & -7.60 & 35.93 & -6.84 & 35.41 & -8.19 \\
\hline
\end{tabular}

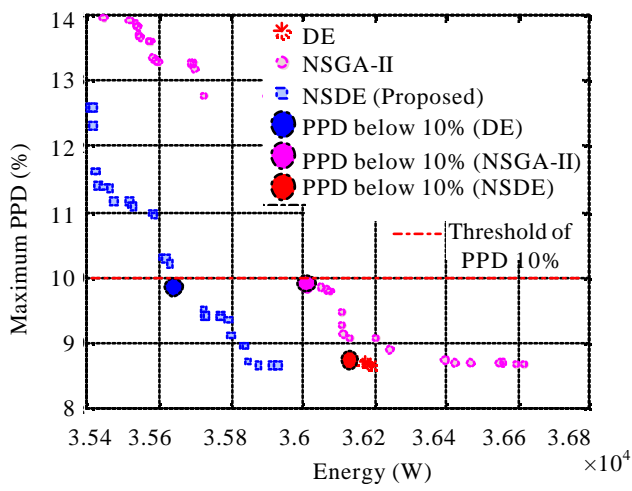

Fig. 9: Selected Pareto fronts for decision making process (PPD below 10\%)

respectively, compared to the baseline scenario. It can be observed that the proposed NSDE shows the highest energy saving in this configuration (Table 5).

In the next configuration, the extreme points of Pareto curve are examined. At one extreme point, thermal comfort is given the highest priority thus Pareto solution with the lowest $\mathrm{PPD}^{\max }$ is selected for energy performance evaluation. The selected Pareto fronts are highlighted in Fig. 10. The energy consumption of DE, NSGA-II and NSDE are found to be at $36.19,36.62$ and $35.93 \mathrm{~kW}$, respectively. Compared to the baseline scenario, this shows an energy saving of $6.17,5.06$ and $6.84 \%$, respectively. As a result, the proposed NSDE still yields the highest energy reduction among the 3 algorithms.

At another extreme point, thermal comfort is given the lowest priority where Pareto solution with the highest $\mathrm{PPD}^{\max }$ is chosen for investigation. The energy consumption of DE, NSGA-II and NSDE show readings of $36.13,35.45$ and $35.41 \mathrm{~kW}$, respectively which correspond to energy reduction of $6.33,8.09$ and $8.19 \%$ compared to the baseline scenario with no optimization.

Table 5 summarises the results of energy consumption of various algorithms and the energy differences compared to baseline scenario. As a conclusion all three algorithms show energy saving compared to baseline scenario case. However, the proposed NSDE shows the highest energy saving characteristic in all the three configurations examined.

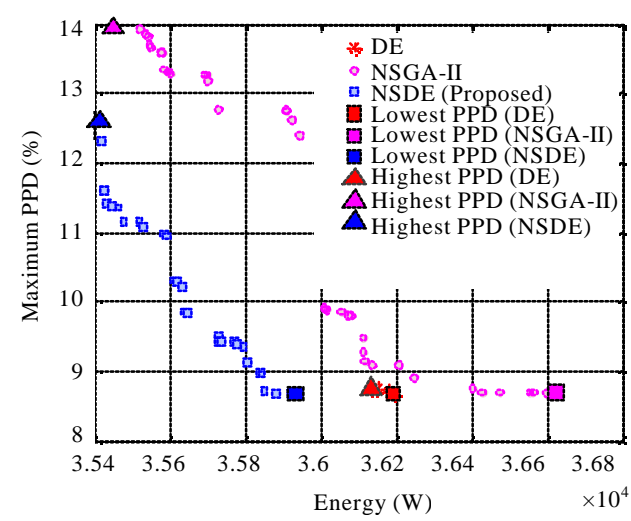

Fig. 10: Selected Pareto fronts for decision making process (Lowest PPD and highest PPD)

This proves that NSDE has the best optimality solutions at every weights of preference assignment in decision making and has the best energy saving performance of all the algorithms investigated. We can also observed that if occupants prioritize energy saving than thermal comfort, NSDE can achieve even more saving at $8.2 \%$ with a little compromise to thermal comfort with PPD of $12.6 \%$ (Fig. 10).

\section{CONCLUSION}

This study proposed a multi-objective optimization approach based on Non-dominated Sorting Genetic Algorithm-II (NSGA-II) and Differential Evolution (DE) algorithms. The proposed NSDE incorporates the efficient searching ability of DE and fast Pareto optimal solutions sorting of NSGA-II in solving for multi-objective optimization of HVAC system. The optimization is performed using MATLAB and EnergyPlus in searching for the optimal cooling temperature set points so that the best trade-off solutions between energy consumption and thermal comfort can be obtained.

The results of the optimization are compared with the base algorithms using some standard performance metrics which quantify the cardinality, space and diversity of the obtained Pareto fronts. The proposed NSDE shows a better convergence, diversity and distribution of Pareto fronts compared to NSGA-II and DE through evaluation of Spacing metric (S) and Hyper-Volume (HV) metrics. As for cardinality measure, although, NSGA-II produces 
higher number of non-dominated solutions in the optimization run, the number of solutions found by NSDE is still considerably high.

Decision making is carried out to compare the energy efficiency of various algorithms using a single solution in the Pareto curve. All optimization results are benchmarked with a baseline scenario where no optimization is involved. Optimization results show that the proposed NSDE yields the highest energy saving in comparison to the baseline scenario among the three algorithms examined. The energy reduction range from $6.8-8.2 \%$ depends on different weightage of preference assignment. This study investigates optimization using a single day of the hottest month. The proposed algorithm is expected to result in more energy saving if it is applied to the HVAC systems throughout the year.

\section{RECOMMEDATIONS}

It can be concluded that the proposed NSDE shows potential for the solution of multi-objective optimization in HVAC systems. It would be interesting as a future research to investigate the energy performance of the algorithm throughout the year to see a more significant energy saving potential. Future research will also be dedicated to benchmark the energy and thermal comfort profile of the proposed algorithm against the popular swarm intelligence algorithms such as PSO and Ant colony.

\section{REFERENCES}

ANSI/ASHRAE., 2013. Thermal environmental conditions for human occupancy standard 55-2013. American Society of Heating, Refrigerating and Air-Conditioning Engineers, Inc., Atlanta, Georgia.

ASHRAE., 2009. ASHRAE Handbook of Fundamentals. Vol. 30329, ASHRAE, Akron, Ohio, Pages: 544.

Anonymous, 2013. Multi-objective performance metrics. Department of Electrical Engineering, Faculty of Engineering, Dayalbagh Educational Institute, Agra, India.http://shodhganga.inflibnet.ac.in/bitstream/ 10603/15070/28/28_appendix_h.pdf

Awad, M. and R. Khanna, 2015. Multiobjective Optimization. In: Efficient Learning Machines: Theories, Concepts and Applications for Engineers and System Designers, Awad, M. and R. Khanna (Eds.). Apress, New York, USA., ISBN-13:9781-4302-5989-3, pp: 185-208.

Beghi, A., L. Cecchinato and M. Rampazzo, 2011. A multi-phase genetic algorithm for the efficient management of multi-chiller systems. Energy Convers. Manage., 52: 1650-1661.
Beghi, A., L. Cecchinato, G. Cosi and M. Rampazzo, 2012. A PSO-based algorithm for optimal multiple chiller systems operation. Appl. Therm. Eng., 32: 31-40.

Chiandussi, G., M. Codegone, S. Ferrero and F.E. Varesio, 2012. Comparison of multi-objective optimization methodologies for engineering applications. Comput. Math. Appl., 63: 912-942.

Coelho, L.D.S. and A. Askarzadeh, 2016. An enhanced bat algorithm approach for reducing electrical power consumption of air conditioning systems based on differential operator. Appl. Therm. Eng., 99: 834-840.

Coelho, L.D.S. and V.C. Mariani, 2013. Improved firefly algorithm approach applied to chiller loading for energy conservation. Energy Build., 59: 273-278.

Deb, K., A. Pratap, S. Agarwal and T. Meyarivan, 2002. A fast and elitist multiobjective genetic algorithm: NSGA-II. IEEE Trans. Evol. Comput., 6: 182-197.

Delgarm, N., B. Sajadi, F. Kowsary and S. Delgarm, 2016. Multi-objective optimization of the building energy performance: A simulation-based approach by means of Particle Swarm Optimization (PSO). Appl. Energy, 170: 293-303.

Fong, K.F., V.I. Hanby and T.T. Chow, 2006. HVAC system optimization for energy management by evolutionary programming. Energy Build., 38: 220-231.

Fong, K.F., V.I. Hanby and T.T. Chow, 2009. System optimization for HVAC energy management using the robust evolutionary algorithm. Appl. Therm. Eng., 29: 2327-2334.

Garnier, A., J. Eynard, M. Caussanel and S. Grieu, 2015. Predictive control of multizone heating, ventilation and air-conditioning systems in non-residential buildings. Appl. Soft Comput., 37: 847-862.

He, X., Z. Zhang and A. Kusiak, 2014. Performance optimization of HVAC systems with computational intelligence algorithms. Energy Build., 81: 371-380.

Huang, W. and H.N. Lam, 1997. Using genetic algorithms to optimize controller parameters for HVAC systems. Energy Build., 26: 277-282.

Hussain, S., H.A. Gabbar, D. Bondarenko, F. Musharavati and S. Pokharel, 2014. Comfort-based fuzzy control optimization for energy conservation in HVAC systems. Control Eng. Pract., 32: 172-182.

Kusiak, A., G. Xu and F. Tang, 2011. Optimization of an HVAC system with a strength multi-objective particle-swarm algorithm. Energy, 36: 5935-5943.

Kusiak, A., M. Li and F. Tang, 2010. Modeling and optimization of HVAC energy consumption. Applied Energy, 87: 3092-3102. 
Lee, W.S., Y.T. Chen and Y. Kao, 2011. Optimal chiller loading by differential evolution algorithm for reducing energy consumption. Energy Build., 43: 599-604.

Magnier, L. and F. Haghighat, 2010. Multiobjective optimization of building design using TRNSYS simulations, genetic algorithm and artificial neural network. Build. Environ., 45: 739-746.

Mezura-Montes, E., M. Reyes-Sierra and C.A.C. Coello, 2008. Multi-Objective Optimization Using Differential Evolution: A Survey of the State-of-the-Art. In: Advances in Differential Evolution, Chakraborty, U.K., (Eds.). Springer, Berlin, Germany, ISBN: 978-3-540-68827-3, pp: 173-196.

Mossolly, M., K. Ghali and N. Ghaddar, 2009. Optimal control strategy for a multi-zone air conditioning system using a genetic algorithm. Energy, 34: 58-66.

Nassif, N., S. Kajl and R. Sabourin, 2004. Evolutionary algorithms for multi-objective optimization in HVAC system control strategy. Proceedings of the IEEE Annual Conference on Fuzzy Information, Processing Vol. 1 (NAFIPS'04), June 27-30, 2004, IEEE, Banff, Alberta, Canada, pp: 51-56.

Riquelme, N., C. von Lucken and B. Baran, 2015. Performance metrics in multi-objective optimization. Proceedings of the 2015 Latin American Computing Conference (CLEI), October 19-23, 2015, IEEE, Arequipa, Peru, pp: 1-11.

Seo, J., R. Ooka, J.T. Kim and Y. Nam, 2014. Optimization of the HVAC system design to minimize primary energy demand. Energy Build., 76: 102-108.

Srinivas, N. and K. Deb, 1994. Multiobjective optimization using nondominated sorting in genetic algorithms. Evol. Comput., 2: 221-248.
Storn, R. and K. Price, 1997. Differential evolution-A simple and efficient heuristic for global optimization over continuous spaces. J. Global Optim., 11: 341-359.

Tydrichova, M. and W. Kozlowska, 2017. Analysis of various multi-objective optimization evolutionary algorithms for monte carlo treatment planning system. MSc Thesis, European Organization for Nuclear Research, Geneva, Switzerland.

Wang, S. and X. Jin, 2000. Model-based optimal control of VAV air-conditioning system using genetic algorithm. Build. Environ., 35: 471-487.

Wright, J.A., H.A. Loosemore and R. Farmani, 2002. Optimization of building thermal design and control by multi-criterion genetic algorithm. Energy Build., 34: 959-972.

$\mathrm{Xu}, \mathrm{X}$. and S. Wang, 2009. A model-based optimal ventilation control strategy of multi-zone VAV air-conditioning systems. Applied Thermal Eng., 29: 91-104.

Xu, Y., K. Ji, Y. Lu, Y. Yu and W. Liu, 2013. Optimal building energy management using intelligent optimization. Proceedings of the 2013 IEEE International Conference on Automation Science and Engineering (CASE'13), August 17-20, 2013, IEEE, Madison, Wisconsin, ISBN:978-1-4799-1515-6, pp: 95-99.

Yang, R. and L. Wang, 2012. Multi-objective optimization for decision-making of energy and comfort management in building automation and control. Sustainable Cities Soc., 2: 1-7.

Zeng, Y., Z. Zhang and A. Kusiak, 2015. Predictive modeling and optimization of a multi-zone HVAC system with data mining and firefly algorithms. Energy, 86: 393-402.

Zheng, G.R. and M. Zaheer-Uddin, 1996. Optimization of thermal processes in a variable air volume HVAC system. Energy, 21: 407-420. 